\title{
Sheng-Mai San
}

National Cancer Institute

\section{Source}

National Cancer Institute. Sheng-Mai San. NCI Thesaurus. Code C102756.

A Chinese herbal medicine composed of extracts from the roots of Panax Ginseng (ren shen) and Ophiopogon japonicas (mai men dong), and the berries of Schisandra chinensis (wu wei zi) with potential protective activity. Ginseng contains a complex mixture of saponins, ginsenosides and panaxosides; homoisoflavonoids isolated from Ophiopogon show anti-inflammatory properties; Schisandra contains abundant amounts of phytoestrogen lignans with antioxidant activity. Although the mechanism of action maybe inexplicit or complex, these phytochemicals work synerg istically and may improve symptoms such as shortness of breath, sweating, cough, thirst, dry mouth, and palpitations as well as have an effect on chemotherapy or radiotherapy-induced fatigue, weakness, and neutropenia. 